\title{
Combining 2D and 3D Device Simulation with Circuit Simulation for Optimising High-Efficiency Silicon Solar Cells
}

\author{
Gernot Heiser ${ }^{a}$, Pietro P. Altermatt ${ }^{b}$, James Litsios $^{c}$ \\ ${ }^{a}$ School of Computer Science and Engineering, \\ The University of New South Wales, Sydney 2052, AUSTRALIA \\ ${ }^{b}$ Centre for Photovoltaic Devices and Systems, UNSW, Sydney 2052, AUSTRALIA \\ ${ }^{c}$ Integrated Systems Lab, ETH-Zurich, 8092 Zurich, SWITZERLAND
}

\begin{abstract}
This paper reports on simulation techniques developed for the modelling and optimisation of complete, $2 \times 2 \mathrm{~cm}^{2}$, high-efficiency silicon solar cells. We use three-dimensional (3d) device simulation to extract a J-V curve of an interior section of a cell. 2D simulations of the cell perimeter are then used to correct the J-V curves for the loss of carriers across the cell boundary. The resulting characteristics are input to a circuit simulation which connects the various cell sections into a model of a full cell. The J-V curve which results from that simulation can be directly compared to measured data. We find excellent agreement between simulation and measurement.
\end{abstract}

\section{Introduction}

The passivated emitter, rear locally diffused (PERL) silicon solar cell (Fig. 1), developed at the University of New South Wales (UNSW), features the highest independently confirmed efficiency, $24.0 \%$, of any silicon solar cell under unconcentrated terrestrial illumination conditions [1]. The goal at UNSW is to improve PERL cell efficiency to $25 \%$.

In the last two years $2 \mathrm{~d}$ device simulation has been used extensively at UNSW to characterise PERL cells and has lead to a detailed understanding of the effects limiting internal cell efficiency [2]. A first 3d study [3] performed a year ago showed that $3 \mathrm{~d}$ effects, resulting from the point contact pattern at the rear of the cell (Fig. 1), do not have a significant effect on efficiency if a single irreducible section of the cell (see below) is considered in isolation. However, a detailed examination of the losses due to a cell's serial resistance, $R_{s}$, showed that losses resulting from the resistivity of the front contact grid (Fig. 2) were larger than anticipated [4]. These losses are mostly a result of the so-called non-generation loss: Owing to the voltage drop along the contact grid, different parts of the cell operate under slightly different bias. Therefore, not all parts of the cell can operate under maximum power point (MPP) conditions at a fixed external bias, resulting in a degradation of conversion efficiency. This effect had previously been underestimated. The study, which was based on a combination of 


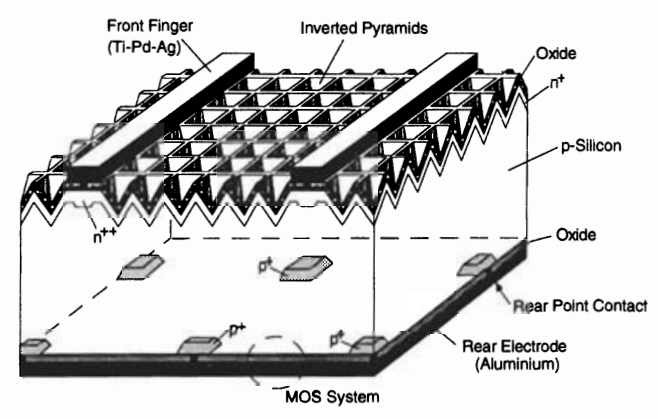

Figure 1: The UNSW PERL Si solar cell (partial view).

measurements and simulation, has led to a change in the front contact grid geometry, which contributed significantly to the latest efficiency improvement from $23.5 \%$ to $24.0 \%$.

From this experience it is obvious that in order to obtain a fully optimised PERL design it is necessary to model the cell in its entirety. So far our $3 \mathrm{~d}$ simulations were restricted to the irreducible section given as 'waver thickness' $\times$ 'half the front contact grid spacing' $\times$ 'half the rear point contact spacing', $370 \mu \mathrm{m} \times 400 \mu \mathrm{m} \times$ $133 \mu \mathrm{m}$ for a typical PERL cell. This is sufficient under the assumption of negligible metal resistance and negligible perimeter effects.

While the study by Altermatt [4] showed that the first of these assumptions is not valid, the second one is not valid either. We know from simplified analytical models that perimeter effects degrade the MPP voltage by about $4 \mathrm{mV}$ [2], a small but noticeable effect. However, no detailed studies of perimeter effects in solar cells have been reported to date.
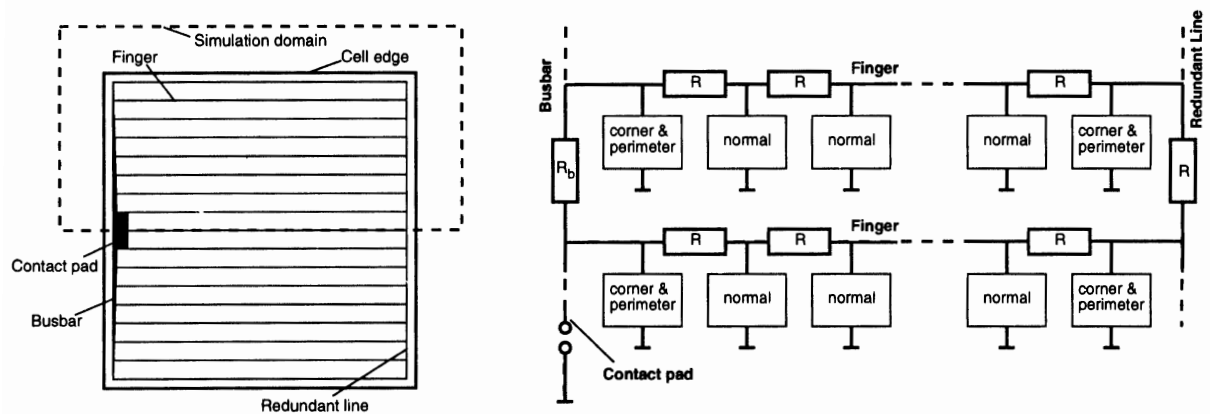

Figure 2: Schematic view of the front contact pattern of a PERL cell (left) and equivalent circuit (right).

\section{Full Cell Simulations}

As a full $3 \mathrm{~d}$ simulation of a PERL cell is beyond the limits of present hardware and simulation technology, we chose an approach which combines device with circuit simulation. A J-V curve is extracted from a standard $3 \mathrm{~d}$ simulation of an irreducible 


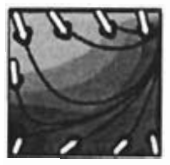

Figure 3: Comparison of $2 \mathrm{~d}$ simulations of unit cell (left) and perimeter region (below), the widths of the simulation domains are $400 \mu \mathrm{m}$ and $3067 \mu \mathrm{m}$ respectively. The region covered by the unit cell simulation corresponds to the leftmost part of the perimeter simulation. The plots show electron current density (light colours imply strong current) and electron current flow at MPP conditions.

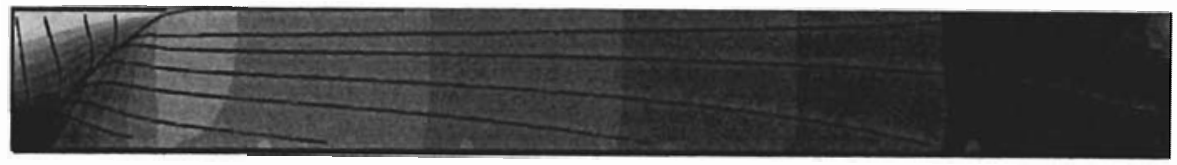

section of the cell. The whole cell can be thought as consisting of many of these unit cells, connected by the metal contacts. Under the assumption of negligible current flow across the boundaries of the unit cells, the effect of the metal can be lumped into ohmic resistors connecting the unit cells (Fig. 2). We can then model the operation of the whole cell using a circuit simulation.

The intersections between the contact fingers and the collecting busbar, or the redundant line at the opposite end (cf. Fig. 2), require special treatment, as these areas cannot be reasonably modelled by one of our unit cells. We therefore performed a separate $3 \mathrm{~d}$ simulation to yield a $\mathrm{J}-\mathrm{V}$ curve of such corner cells.

The perimeter regions present a different problem: To avoid efficiency losses resulting from the damage created by sawing, the cells remain embedded in the wafer for measurement. An accurate model needs to account properly for the loss of carriers across the edge of the cell. Due to the huge minority carrier diffusion lengths (several $\mathrm{mm}$ in PERL cells) the active perimeter region is quite large, too large for a $3 \mathrm{~d}$ simulation. However, since the perimeter region is essentially two dimensional, $2 \mathrm{~d}$ simulations are adequate for the determination of the effect of the perimeter region. To this end we perform a $2 \mathrm{~d}$ simulation of the perimeter region, as well as a $2 \mathrm{~d}$ simulation of the unit cell. We take the difference of the two J-V curves to represent the perimeter effect. This is then added to the $\mathrm{J}-\mathrm{V}$ curve of a unit cell to yield a $3 \mathrm{~d}$ result which is corrected for perimeter effects; such a corrected unit cell can then be used to represent a section of the edge region of the PERL cell. The current densities across the perimeter are several orders of magnitude smaller than the current densities within the illuminated area, hence errors introduced by this correction scheme are small compared to the correction itself.

A typical PERL cell has a finger spacing of $800 \mu \mathrm{m}$ and a rear contact spacing of $250 \mu \mathrm{m}$. With a total size of $2 \times 2 \mathrm{~cm}^{2}$, the cell can be thought to consist of approx. 1000 unit cells, which, together with a similar number of connecting resistors, is quite a large number of devices for a circuit, simulation. However, it is not necessary to simulate that many devices. The internal unit cells along a contact finger are all identical and several of them can be lumped into a single device (properly scaled). We found that 10 internal unit cells per finger were sufficient to obtain a converged result. With 13 fingers (for symmetry reasons only half the cell needs to be simulated, cf. Fig. 2) this leads to a circuit comprising 156 unit solar cells and a similar number of resistors.

\section{Implementation and Results}

In order to simplify the feeding of device simulation results into the subsequent circuit simulation, we extended the mixed-mode device and circuit simulator DESSIS-ISE [5] 
by a new circuit device type, vi, which is a voltage-controlled current source. This device is defined by an I-V curve in tabular form, which we obtain as an output from our device simulations, also performed using DESSIS-ISE. We therefore require the following for the simulation of a full PERL cell: one $2 \mathrm{~d}$ unit cell simulation (5k grid points, $20 \mathrm{~min}$ CPU time), one $2 \mathrm{~d}$ perimeter simulation $(25 \mathrm{k}, 3 \mathrm{~h})$, one $3 \mathrm{~d}$ unit cell simulation $(65 \mathrm{k}, 12 \mathrm{~h})$, and one $3 \mathrm{~d}$ corner cell simulation $(110 \mathrm{k}, 24 \mathrm{~h})$, plus the circuit simulation $(312$ circuit elements, $1 \mathrm{~h}$ ). CPU times quoted are for a full $\mathrm{J}-\mathrm{V}$ curve on a $60 \mathrm{MHz}$ Sun SS-20. To determine $R_{s}$ we require a second J-V curve for a slightly different light intensity [4].

Fig. 4 shows the simulated voltage profile of a PERL cell as compared to measurement. The model parameters for the simulations where based on experimentally determined values wherever possible. The voltage profiles are clearly in excellent agreement. An extensive parameter study with the aim of optimising the contact grid design is presently under way.
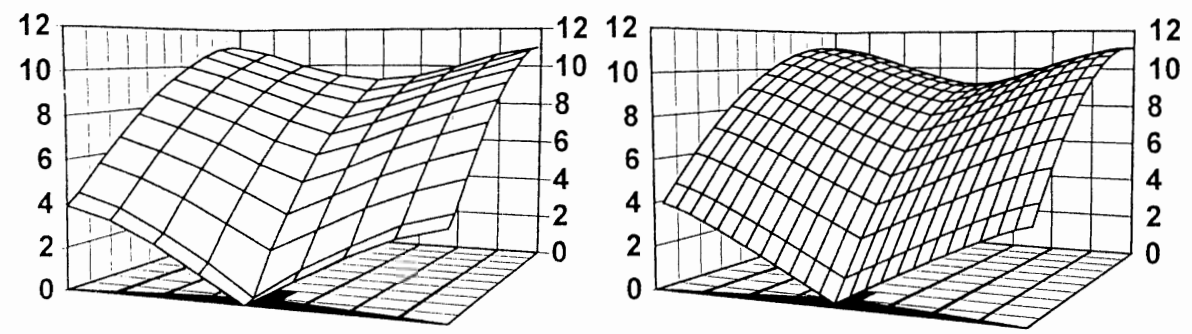

Figure 4: Measured (left) and simulated (right) voltage profile $[\mathrm{mV}]$ of the front contact grid of a PERL cell.

\section{Acknowledgement}

This work is supported by a grant from the Australian Research Council.

\section{References}

[1] J. Zhao, A. Wang, P. P. Altermatt, S. R. Wenham, and M. A. Green. $24 \%$ Efficient silicon solar cells. In 1st World Conf. Photovoltaic Energy Conversion, Waikoloa, Hawaii, USA, December 1994. IEEE. To be published.

[2] A. G. Aberle, P. P. Altermatt, G. Heiser, S. J. Robinson, A. Wang, J. Zhao, U. Krumbein, and M. A. Green. Limiting loss mechanisms in 23-percent efficient silicon solar cells. J. Appl. Physics, 1995. To appear.

[3] G. Heiser and A. G. Aberle. Numerical modelling of non-ideal current-voltage characteristics of high-efficiency silicon solar cells. In 5th Int. W. Num. Modeling Proc. and Dev. for Integr. Circ., pages 177-80, Honolulu, USA, June 1994. IEEE.

[4] P. P. Altermatt. Two-dimensional numerical modelling of high-efficiency silicon solar cells. Diploma thesis (physics), University of Constance, Germany, July 1994. Thesis project carried out at UNSW, Sydney, Australia.

[5] ISE Integrated Systems Engineering AG, Zurich, Switzerland. DESSIS 1.3.6: Manual, 1994. 